\title{
Numerical homogenization of non linear composites with a polarization-based FFT iterative scheme
}

\author{
Vincent MONCHIET, Guy BONNET \\ Université Paris-Est, Laboratoire Modélisation et Simulation Multi Echelle, MSME \\ UMR8208 CNRS, 5 boulevard Descartes, 77454 Marne la Vallée Cedex, France
}

\begin{abstract}
This paper presents an extension of the polarization based iterative scheme in the context of non linear composites. The polarization based algorithm, used for computing the effective properties of composite materials, converges independently of the value of the contrast between the phases. However the application of this scheme in the initial form is restricted to the case of linear elasticity. In this paper we propose some modifications of this iterative scheme in order to deal with the most general case of non linear constituents. Two applications are proposed to show the capability of the method. The first concerns a composite made up of a power-law type non linear matrix with rigid inclusions while in the second illustration we deals with the determination of the macroscopic yield surface of porous plastic materials.
\end{abstract}

Key words: Non Linear Composites, Homogenization, Fast Fourier Transform, Polarization, Power law material analysis

\section{Introduction}

Since two decades, the Fast Fourier based iterative schemes has been employed for various applications in the field of homogenization of periodic microstructures (see for instance Bilger et al. [2], Willot et al. [28], Brenner et al. [5], Brenner [4], Monchiet and Bonnet [18], Lee et al. [13], Ghossein and Lévesque [10], Li et al. [14], Lebensohn [12], Nguyen et al. [22]...). The FFT based iterative scheme has been proposed, in its simplest form, by Moulinec and Suquet

Email addresses: vincent.monchiet@univ-paris-est.fr (Vincent MONCHIET), guy. bonnet@univ-paris-est.fr (Guy BONNET). 
[20] and generalized later for non linear composites [21]. The method of resolution uses exact expressions of the Green operator in the Fourier space and the Fast Fourier Transform (FFT) algorithm ${ }^{1}$. It is an efficient alternative to micromechanical Finite Element methods since it considers the exact images of the microstructures which can be obtained, in particular, from modern experimental techniques such as tomography. However, the rate of convergence of this algorithm initially proposed in $[20,21]$ is known to depend drastically on the contrast between the phases and cannot be used for infinite contrasts. To circumvent these incapacities, new iterative schemes, based on the augmented Lagrangian, have been further proposed $[15,16]$. Note also that in [27], the authors provide an extension of the "accelerated" scheme first proposed by Eyre and Milton [7] for applications to nonlinear periodic composites. Alternatively, in [17], we have proposed a polarization based iterative scheme for computing the macroscopic properties of elastic composites. The method uses the same ingredients as the strain based iterative schemes, proposed by Moulinec and Suquet [20] and its dual (stress based) formulation [1,3]: the periodic Green tensors for the strain and the stress, the FFT algorithm for computing the Fourier Transform and its inverse... No additional treatment or numerical tools (Lagrangian, conjugate gradient,...) are required for its implementation. The approach has the particularity to solve the unit cell problem for the elastic composites for which an average polarization is prescribed over the unit cell instead of the usual average macroscopic strain or stress in the classical approaches. It has been shown through several numerical tests that the convergence of this new iterative scheme is independent of the local elastic properties. More specifically, it has been shown that the problem of composites containing both voids and rigid inclusions can be solved by the method with a very good rate of convergence. By taking advantage of the linearity of the considered elasticity equations, it is possible to obtain the effective properties by computing the macroscopic strain and stress for a prescribed average polarization.

In this paper we provide an extension of the polarization approach to the case of non linear composites. The initial polarization scheme has a specificity since it provides the solution of a problem related to a unit cell submitted to an average polarization. For applications to non linear composites it is practically necessary to consider the unit cell problem submitted to an average macroscopic strain or stress instead of an average polarization. Indeed, two scales homogenization methods in the context of non linear structure problems (see for example $[25,8,9]$ ), considered a macroscopic strain applied to the RVE in order to compute the macroscopic response of the composites.

The paper is organized in three parts. In section 2, we recall the cell problem in the context of elasticity and the polarization based iterative scheme for its solution. Still in the context of elastic constituents, we propose in sec-

\footnotetext{
1 Note that an alternative method which does not use Fourier Transform and its inverse has been recently proposed by Yvonnet [29]
} 
tion 3 to modify this algorithm in order to deal with prescribed macroscopic strain or stress. The case of non linear composites is considered in section 4 . The method is implemented for a composite made up of a power-law type non linear matrix with rigid inclusions. Various calculations based on several microstructure occurrences are then performed to show the good rate of convergence of the polarization based iterative scheme. In a second illustration, we determine the macroscopic yield surface of plastic materials which contains randomly distributed cylindrical cavities.

\section{The polarization based iterative scheme for solving the unit cell elastic problem}

Let us recall the elastic problem for the unit cell of the periodic composite:

$$
\left\{\begin{array}{l}
\boldsymbol{\varepsilon}(\underline{x})=\frac{1}{2}\left(\nabla \underline{u}(\underline{x})+\nabla^{t} \underline{u}(\underline{x})\right), \quad \forall \underline{x} \in V \\
\boldsymbol{\sigma}(\underline{x})=\mathbb{C}(\underline{x}): \boldsymbol{\varepsilon}(\underline{x}), \quad \forall \underline{x} \in V \\
\operatorname{div}(\boldsymbol{\sigma}(\underline{x}))=0, \quad \forall \underline{x} \in V \\
\underline{u}(\underline{x})-\boldsymbol{E} \cdot \underline{x} \text { periodic } \\
\boldsymbol{\sigma}(\underline{x}) \cdot \underline{n} \text { antiperiodic }
\end{array}\right.
$$

in which $\underline{u}(\underline{x}), \varepsilon(\underline{x}), \boldsymbol{\sigma}(\underline{x})$ are the local displacement, strain and stress field respectively. By $\boldsymbol{E}$ we denote the macroscopic strain and by $\mathbb{C}(\underline{x})$ the local elastic tensor which depends on the vector position $\underline{x}$. A parallepiped is considered for the unit cell of the periodic medium (a rectangle for $2 D$ problems) and we denote by $L_{1}, L_{2}$ and $L_{3}$ its lengths taken respectively along the axes $O x_{1}, O x_{2}$ and $O x_{3}$ of the cartesian coordinate system. A new FFT based iterative scheme has been recently proposed in [17] for the computation of the effective properties of linear elastic composites. This method is formulated with the polarization tensor $\boldsymbol{\tau}(\underline{x})=\left(\mathbb{C}(\underline{x})-\mathbb{C}^{0}\right): \varepsilon(\underline{x})$ and consists in solving the system (1) under prescribed average polarization $\left\langle\boldsymbol{\tau}(\underline{x})>_{V}=\boldsymbol{T}\right.$. This new iterative scheme differs from those already proposed in the literature (see [20], [1], [3], [29] etc.) for which the macroscopic strain or stress is prescribed. This algorithm contains 7 steps which are recalled below: 


$$
\begin{array}{ll}
\text { 1) } & \boldsymbol{\tau}^{i}(\underline{x})=\mathcal{F}^{-1}\left(\widehat{\boldsymbol{\tau}}^{i}(\underline{\xi})\right) \\
\text { 2) } & \boldsymbol{\varepsilon}^{i}(\underline{x})=\left(\mathbb{C}(\underline{x})-\mathbb{C}^{0}\right)^{-1}: \boldsymbol{\tau}^{i}(\underline{x}) \\
\text { 3) } & \hat{\boldsymbol{\varepsilon}}^{i}(\underline{\xi})=\mathcal{F}\left(\boldsymbol{\varepsilon}^{i}(\underline{x})\right) \\
\text { 4) } & \hat{\boldsymbol{\sigma}}^{i}(\underline{\xi})=\mathbb{C}^{0}: \widehat{\boldsymbol{\varepsilon}}^{i}(\underline{\xi})+\widehat{\boldsymbol{\tau}}^{i}(\underline{\xi}) \\
\text { 5) } & \text { convergence test } \\
\text { 6) } & \widehat{\boldsymbol{\tau}}^{i+1}(\underline{\xi})=\widehat{\boldsymbol{\tau}}^{i}(\underline{\xi})+\alpha\left[\widehat{\boldsymbol{\Delta}}^{0}(\underline{\xi}): \widehat{\boldsymbol{\varepsilon}}^{i}(\underline{\xi})-\mathbb{C}^{0}: \widehat{\boldsymbol{\Gamma}}^{0}(\underline{\xi}): \widehat{\boldsymbol{\sigma}}^{i}(\underline{\xi})\right], \text { for } \underline{\xi} \neq 0 \\
\text { 7) } & \hat{\boldsymbol{\tau}}^{i+1}(0)=\hat{\boldsymbol{\tau}}^{i}(0), \text { for } \underline{\xi}=0
\end{array}
$$

In the above relations, tensor $\mathbb{C}^{0}$ is the rigidity of the reference medium, which is independent of the vector position. In (2), the symbolic notations $\mathcal{F}$ and $\mathcal{F}^{-1}$ denote respectively the Fourier transform and its inverse. The fourth order tensors $\widehat{\boldsymbol{\Gamma}}^{0}(\underline{\xi})$ and $\widehat{\boldsymbol{\Delta}}^{0}(\underline{\xi})$ are the periodic Green operators for the strain and for the stress. Their exact expression in the Fourier space can be found in [17]. For the numerical implementation of the iterative scheme, a finite number of wave vectors $\underline{\xi}_{n}$ for $n=-N . . N-1$ which are located over the reciprocal lattice.

In the case of an isotropic reference material, the latter is defined by its shear modulus $\mu_{0}$ and its compressibility $k_{0}$. Parameters $\alpha, \mu_{0}$ and $k_{0}$ must be chosen as:

$$
0<\alpha<2, \quad-\infty<\mu_{0}<0, \quad-\infty<k_{0}<0
$$

The inequalities in (3) give then a sufficient condition for having the convergence of the polarization based iterative scheme. It is worth noticing that these conditions of convergence do not depend on the elastic properties of the constituents. The numerical investigations of the rate of convergence of the algorithm (2) has shown that the value $\alpha=3 / 2$ is optimal. The values of $\mu_{0}$ and $k_{0}$ are adjusted in order to obtain the best convergence of the iterative scheme. Their values will be further discussed in the sequel of the paper. At each step of the iterative scheme (2) the condition $\left\langle\boldsymbol{\tau}^{i}(\underline{x})>_{V}=\boldsymbol{T}\right.$ is preserved. The fields $\varepsilon^{i}(\underline{x})$ and $\boldsymbol{\sigma}^{i}(\underline{x})$ are compatible or equilibrated only at convergence of the iterative scheme, namely when $\tau^{i+1}(\underline{x})=\boldsymbol{\tau}^{i}(\underline{x})$. In order to evaluate the accuracy of the solution, we use a test based simultaneously on compatibility and equilibrium:

$$
\max \left(\frac{\sqrt{\sum_{n}\left\|\mathbb{Q}\left(\underline{\xi}_{n}\right): \widehat{\boldsymbol{\varepsilon}}^{i}\left(\underline{\xi}_{n}\right)\right\|^{2}}}{\sqrt{\sum_{n}\left\|\hat{\boldsymbol{\varepsilon}}^{i}\left(\underline{\xi}_{n}\right)\right\|^{2}}}, \frac{\sqrt{\sum_{n}\left\|\mathbb{P}\left(\underline{\xi}_{n}\right): \hat{\boldsymbol{\sigma}}^{i}\left(\underline{\xi}_{n}\right)\right\|^{2}}}{\sqrt{\sum_{n}\left\|\hat{\boldsymbol{\sigma}}^{i}\left(\underline{\xi}_{n}\right)\right\|^{2}}}\right)<\epsilon
$$


The first term, which contains the fourth order tensor $\mathbb{Q}\left(\underline{\xi}_{n}\right)$, is used to check the compatibility for the field $\varepsilon^{i}\left(\underline{\xi}_{n}\right)$ whereas the second term, which contains the fourth order tensor $\mathbb{P}\left(\underline{\xi}_{n}\right)$, corresponds to the equilibrium for $\boldsymbol{\sigma}^{i}\left(\underline{\xi}_{n}\right)$. Tensors $\mathbb{P}(\underline{\xi})$ and $\mathbb{Q}(\underline{\xi})$ are defined by, $\forall \underline{\xi} \neq 0$ :

$$
\mathbb{Q}(\underline{\xi})=(\boldsymbol{I}-\boldsymbol{k}) \underline{\bar{\otimes}}(\boldsymbol{I}-\boldsymbol{k}), \quad \mathbb{P}(\underline{\xi})=\mathbb{I}-\mathbb{Q}(\underline{\xi})
$$

where $\mathbb{I}$ is the identity for fourth order symmetric tensors, $I_{i j k l}=\left(\delta_{i k} \delta_{j l}+\right.$ $\left.\delta_{i l} \delta_{j k}\right) / 2$, the tensorial product $\boldsymbol{a} \bar{\otimes} \boldsymbol{b}$ for second tensors $\boldsymbol{a}$ and $\boldsymbol{b}$ is defined by $(\boldsymbol{a} \underline{\otimes} \boldsymbol{b})_{i j k l}=\left(a_{i k} b_{j l}+a_{i l} b_{j k}\right) / 2$ and the second order tensor $\boldsymbol{k}$ is given by:

$$
\boldsymbol{k}=\frac{1}{|\underline{\xi}|^{2}} \underline{\xi} \otimes \underline{\xi}
$$

\section{The modified iterative scheme}

The iterative process (2) cannot be used in practice for nonlinear constitutive equations, because the mean stresses and strains are obtained at convergence of the algorithm, while in practice these values must be prescribed at the beginning of the iterative process (more particularly in the case of constitutive equations described incrementally). For an extension to non linear composites, we propose to modify the iterative scheme (2) in order to deal with a unit cell with prescribed macroscopic strain $\boldsymbol{E}$. To this end, we replace step 7 in (2) by the following new step:

$$
\left.7^{*}\right) \quad \widehat{\boldsymbol{\tau}}^{i+1}(0)=\widehat{\boldsymbol{\tau}}^{i}(0)+\gamma \mathbb{C}^{0}:\left[<\varepsilon^{i}(\underline{x})>_{V}-\boldsymbol{E}\right]
$$

When the convergence of the new iterative scheme is attained, one has $\widehat{\boldsymbol{\tau}}^{i+1}(0)=$ $\widehat{\boldsymbol{\tau}}^{i}(0)$ and then $\left\langle\boldsymbol{\varepsilon}(\underline{x})>_{V}=\boldsymbol{E}\right.$ which means that the average of the local compatible field $\varepsilon(\underline{x})$ is equal to the prescribed macroscopic strain $\boldsymbol{E}$. In this new iterative scheme, the polarization is still initialized by the constant polarisation $\boldsymbol{T}$ but at convergence, the average of the local polarization, $\boldsymbol{\tau}(\underline{x})$, is no more equal to $\boldsymbol{T}$. In (6), the stiffness tensor $\mathbb{C}^{0}$ is introduced in the last term, so that $\gamma$ is a non dimensional parameter. As concerns the choice of $\gamma$, there is no theoretical arguments that leading to its optimal value, however, several numerical tests have shown that the convergence is reached when $\gamma$ lies in the interval $[0,2]$ and the optimal value is closed to $\gamma=3 / 2$. Note that the parameter $\alpha$ also lies in the interval $[0,2]$ for obtaining the convergence and its optimal value is generally closed to $3 / 2$ (see [17] for more details). Consequently, the value $\gamma=\alpha$ has been considered in our applications. 
Since the equality $<\varepsilon(\underline{x})>_{V}=\boldsymbol{E}$ is only verified at the strict convergence of the iterative scheme, a new condition is added in the convergence test (4). The convergence test of this new algorithm is then:

$$
\max \left(\frac{\sqrt{\sum_{n}\left\|\mathbb{Q}\left(\underline{\xi}_{n}\right): \widehat{\boldsymbol{\varepsilon}}^{i}\left(\underline{\xi}_{n}\right)\right\|^{2}}}{\sqrt{\sum_{n}\left\|\hat{\boldsymbol{\varepsilon}}^{i}\left(\underline{\xi}_{n}\right)\right\|^{2}}}, \frac{\sqrt{\sum_{n}\left\|\mathbb{P}\left(\underline{\xi}_{n}\right): \hat{\boldsymbol{\sigma}}^{i}\left(\underline{\xi}_{n}\right)\right\|^{2}}}{\sqrt{\sum_{n}\left\|\hat{\boldsymbol{\sigma}}^{i}\left(\underline{\xi}_{n}\right)\right\|^{2}}},\right.
$$

Alternatively, it is also possible to prescribe the macroscopic stress to the unit cell. In this case the step 7 of the iterative scheme (2) is replaced by:

$$
\left.7^{* *}\right) \quad \widehat{\boldsymbol{\tau}}^{i+1}(0)=\widehat{\boldsymbol{\tau}}^{i}(0)+\delta\left[<\boldsymbol{\sigma}^{i}(\underline{x})>_{V}-\boldsymbol{\Sigma}\right]
$$

When the convergence is reached, the average of $\boldsymbol{\sigma}(\underline{x})$ complies with $<\boldsymbol{\sigma}(\underline{x})>_{V}=$ $\Sigma$. The value $\delta=-\alpha$ has been found to be optimal in our calculations and is considered in the next part of the paper. Again a constant polarisation $\boldsymbol{T}$ is used to initialize the iterative scheme. The convergence test used for this iterative scheme is:

$$
\max \left(\frac{\sqrt{\sum_{n}\left\|\mathbb{Q}\left(\underline{\xi}_{n}\right): \widehat{\boldsymbol{\varepsilon}}^{i}\left(\underline{\xi}_{n}\right)\right\|^{2}}}{\sqrt{\sum_{n}\left\|\widehat{\boldsymbol{\varepsilon}}^{i}\left(\underline{\xi}_{n}\right)\right\|^{2}}}, \frac{\sqrt{\sum_{n}\left\|\mathbb{P}\left(\underline{\xi}_{n}\right): \widehat{\boldsymbol{\sigma}}^{i}\left(\underline{\xi}_{n}\right)\right\|^{2}}}{\sqrt{\sum_{n}\left\|\hat{\boldsymbol{\sigma}}^{i}\left(\underline{\xi}_{n}\right)\right\|^{2}}},\right.
$$

To summarize, we now have three iterative schemes corresponding respectively to:

- a unit cell subjected to an average polarization $\boldsymbol{T}$. This is the original approach developed in [17] and given by (2). This scheme is called Tpolarization scheme in the sequel of the paper.

- a unit cell submitted to a macroscopic strain $\boldsymbol{E}$, for which step 7 in (2) is replaced by (6). This new algorithm is called thereafter E-polarization scheme.

- a unit cell submitted to a macroscopic strain $\boldsymbol{\Sigma}$, for which step 7 in (2) is replaced by (8). This approach is called $\Sigma$-polarization scheme. 
In order to validate the substantial modifications of the initial polarization based iterative scheme, we propose to show that the rate of convergence of the E-polarization and $\Sigma$ polarization schemes is as good as the initial formulation proposed in [17]. For this, we consider a linear elastic composite consisting of a matrix containing randomly distributed cylindrical inclusions. The cell, depicted on figure 1, is assumed to be subjected to plane strain loadings. The matrix and the inclusion are both incompressible and we denote by $\mu_{M}$ and $\mu_{I}$ their shear moduli. The cell contains 25 inclusions having the radius $R=0.05$ (the cell is squared and its area is equal to 1 ): the volume fraction of inclusions is then $c_{I}=0.196$. We now examine the rate of convergence of the three polarization based iterative schemes for various values of the contrast $\mu_{I} / \mu_{M}$. The unit cell is submitted to the macroscopic loadings $\left(T_{11}=-T_{22}=1\right.$ and $\left.T_{12}=0\right)$ for the T-polarization scheme, to $\left(E_{11}=-E_{22}=1\right.$ and $\left.E_{12}=0\right)$ for the E-polarization scheme and to $\left(\Sigma_{11}=-\Sigma_{22}=1\right.$ and $\left.\Sigma_{12}=0\right)$ for the $\Sigma$-polarization scheme. Each iterative scheme is initialized by the polarization $T_{11}=-T_{22}=1$ and $T_{12}=0$. The modulus of the reference medium is chosen as $\mu_{0}=-5 \mu_{M}$ when the contrast is larger than 1 . When the contrast is lower than 1 we put $\mu_{0}=-\mu_{M} / 5$ (which is almost optimal for the problem). The value of $\alpha=3 / 2$ has been also considered. The resolution of the image is $512 \times 512$. On figure 2 , we represent the number of iterations at convergence as a function of the contrast obtained for the different polarization schemes. It can be observed that they give comparable results: the convergence is reached for any value of the contrast. Moreover the good rate of convergence obtained with the initial polarization scheme is still observed for the E-polarization scheme and the $\Sigma$-polarization scheme.

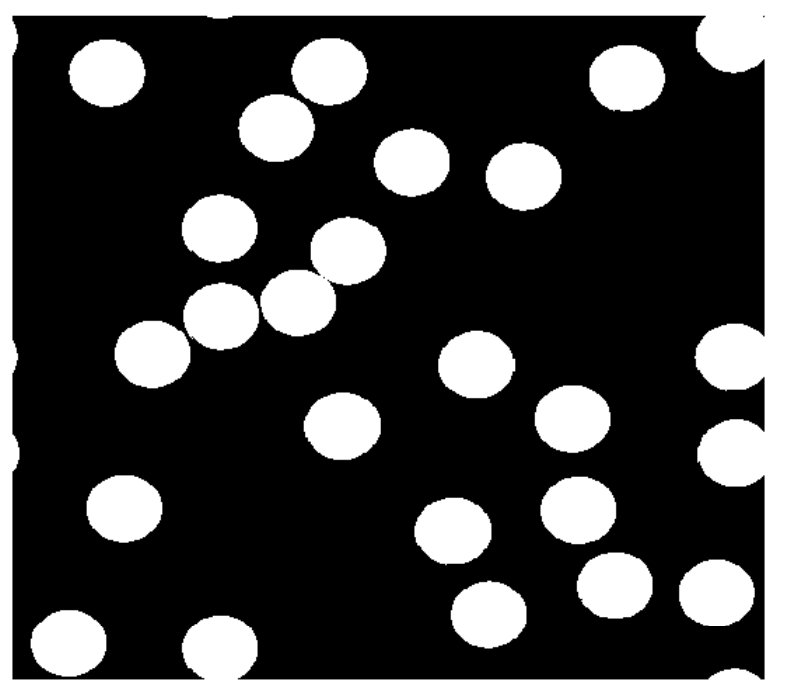

Fig. 1. 


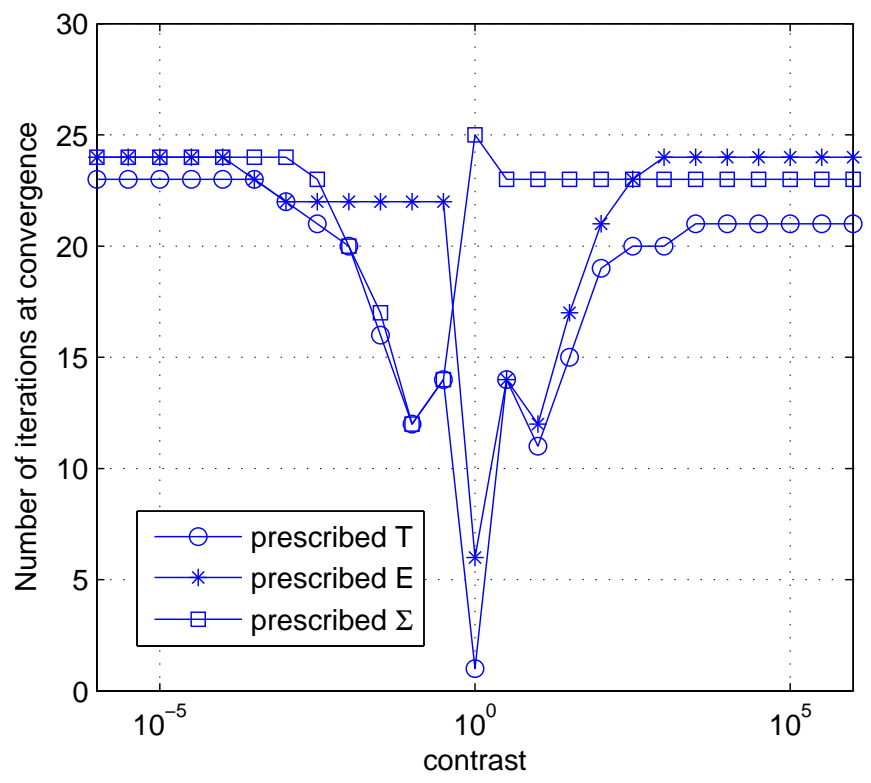

Fig. 2. Number of iterations at convergence as a function of the contrast. Comparison between the initial polarization based iterative scheme (with prescribed average polarization) with the modified schemes which use prescribed average strain $\boldsymbol{E}$ or stress $\boldsymbol{\Sigma}$.

\section{Case of non linear materials}

\subsection{General principles}

We now extend the approach in the case of constituents which obey a non linear behavior. This requires a modification of step 2 in (2). The strain-stress relation can be put into the general form:

$$
\boldsymbol{\sigma}(\underline{x})=\boldsymbol{F}(\varepsilon(\underline{x}))
$$

When the polarization $\boldsymbol{\tau}^{i}(\underline{x})$ is known at step $i$, the associated strain field $\varepsilon^{i}(\underline{x})$ is computed by solving the following non linear equation:

$$
\boldsymbol{F}(\varepsilon(\underline{x}))-\mathbb{C}^{0}: \varepsilon(\underline{x})=\boldsymbol{\tau}^{i}(\underline{x})
$$

at each node of the regular grid. Note that the problem is described here for a non linear elastic law; however, the case of plasticity or viscoplasticity can 
be also considered by using the strain stress relation in an incremental form as already done in [21].

\subsection{Exemple 1: power-law matrix with rigid inclusions}

In this section we propose to investigate the particular case of a power law type material containing rigid inclusions and which has been also considered in the paper of Michel et al. [16]. The matrix is assumed to be incompressible and the deviatoric part of the stress is given by:

$$
\overline{\boldsymbol{\sigma}}(\underline{x})=\frac{2 \sigma_{0}}{3 \varepsilon_{0}}\left(\frac{\varepsilon_{e q}(\underline{x})}{\varepsilon_{0}}\right)^{m-1} \boldsymbol{\varepsilon}(\underline{x}), \quad \operatorname{tr}(\varepsilon(\underline{x}))=0
$$

in which $\varepsilon_{e q}=\sqrt{\frac{2}{3} \varepsilon(\underline{x}): \varepsilon(\underline{x})}$ is the von Mises equivalent strain, $m$ is the powerlaw exponent, $\varepsilon_{0}$ and $\sigma_{0}$ are two material parameters, $\overline{\boldsymbol{\sigma}}(\underline{x})$ is the deviatoric part of the stress tensor $\boldsymbol{\sigma}$. For simplicity we put $\varepsilon_{0}=1$. The non linear equation (11) becomes, for the power-law incompressible material and a linear incompressible reference medium of shear modulus $\mu_{0}$ :

$$
\frac{2 \sigma_{0}}{3}\left(\varepsilon_{e q}(\underline{x})\right)^{m-1} \varepsilon(\underline{x})-2 \mu_{0} \varepsilon(\underline{x})=\bar{\tau}(\underline{x})
$$

where $\overline{\boldsymbol{\tau}}(\underline{x})$ is the deviatoric part of the polarization tensor.

It follows that the equivalent strain, $\varepsilon_{e q}(\underline{x})$, is solution of the non linear equation:

$$
\varepsilon_{e q}^{m}(\underline{x})+p \varepsilon_{e q}(\underline{x})-q=0
$$

with:

$$
p=\frac{3 \mu_{0}}{\sigma_{0}}, \quad q=\frac{\tau_{e q}}{\sigma_{0}}, \quad \tau_{e q}=\sqrt{\frac{3}{2} \overline{\boldsymbol{\tau}}: \overline{\boldsymbol{\tau}}}
$$

Once this non linear equation is solved, the strain field $\varepsilon(\underline{x})$ is given by:

$$
\varepsilon(\underline{x})=\frac{1}{\frac{2 \sigma_{0}}{3}\left(\varepsilon_{e q}(\underline{x})\right)^{m-1}-2 \mu_{0}} \overline{\boldsymbol{\tau}}(\underline{x})
$$

In the rigid inclusion, the strain field is prescribed as zero. Let us recall that the value of the shear modulus of the reference medium, $\mu_{0}$, is chosen strictly 
negative. So, the term " $\frac{2 \sigma_{0}}{3}\left(\varepsilon_{e q}(\underline{x})\right)^{m-1}-2 \mu_{0}$ " which appears in (16) is strictly positive.

The unit cells considered for the applications are represented on figure 3 and consist of randomly distributed rigid circular inclusions. The radius of the inclusions is $R=0.05$ (a squared unit cell with an area equal to 1 is considered for the calculations) and four volume fractions are considered: $c_{I}=0.118$, $c_{I}=0.196, c_{I}=0.275, c_{I}=0.353$. These volume fractions are obtained by considering $M=15, M=25, M=35$ and $M=45$ inclusions in the unit cell. For each volume fraction, various occurrences are also considered.
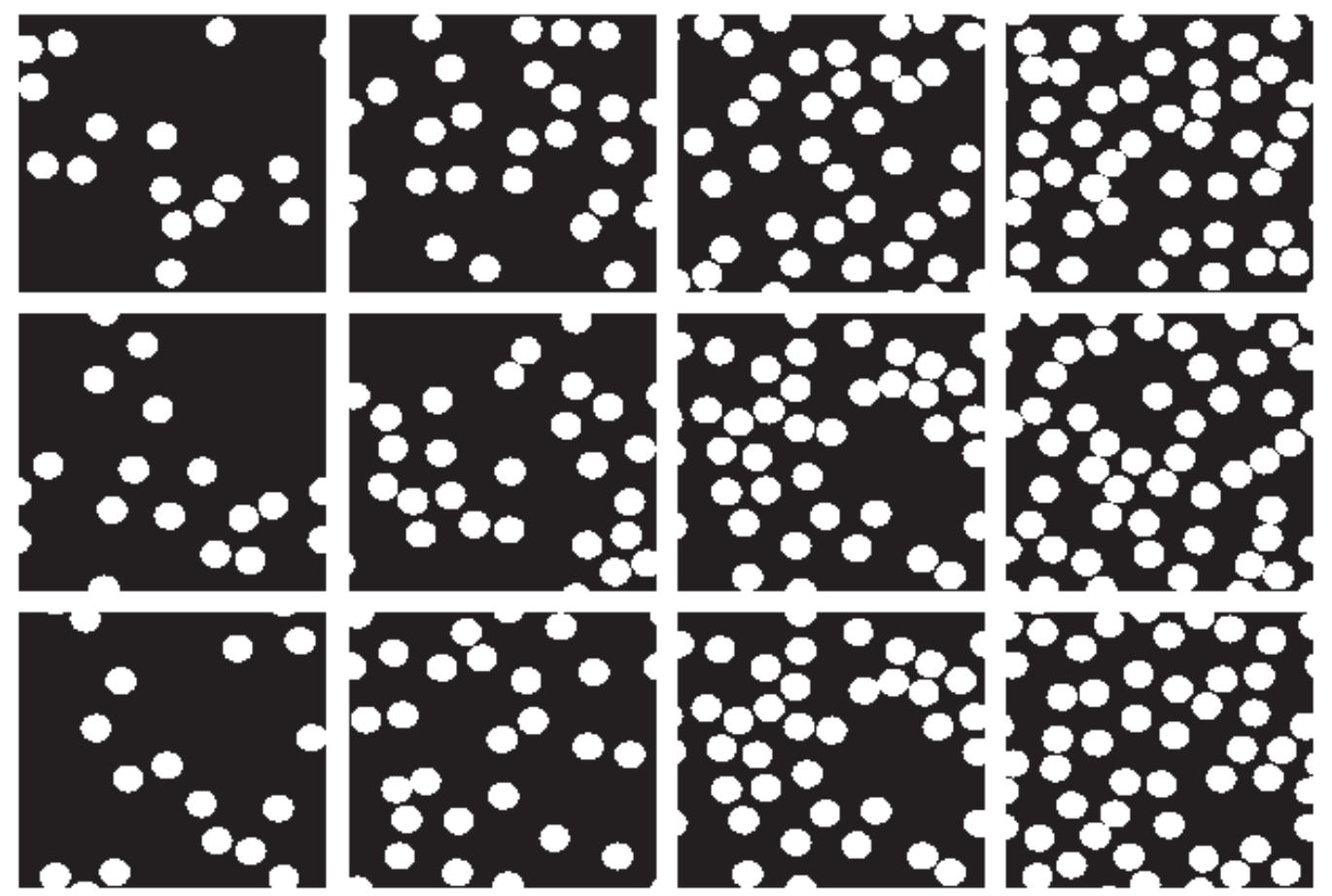

Fig. 3. Unit cells of the periodic composite. From the left to the right, the volume fraction $c_{I}$ of inclusions and the number of inclusions $M$ are: $M=15$ and $c_{I}=0.118$, $M=25$ and $f=0.196, M=35$ and $c_{I}=0.275, M=45$ and $c_{I}=0.353$

For the numerical solution of the cell problems, the E-polarization method is employed. The E-polarization based iterative scheme has been used instead of the $\Sigma$-polarization scheme since for large values of the power-law exponent the behavior of the matrix is closed to a rigid ideally plastic material, and consequently, the value of the macroscopic stress saturates for large values of the macroscopic strain. The cells are then submitted to a macroscopic strain $\boldsymbol{E}$. For our application, the following plane strain conditions are considered: $E_{11}=-E_{22}=1$ and $E_{12}=0$. The iterative scheme is initialized by the uniform polarization $T_{11}=-T_{22}=1$ and $T_{12}=0$. The value $\alpha=3 / 2$ and a regular grid of $512 \times 512$ pixels have been used. There is no theoretical argument which leads 
to the choice of the optimal shear modulus, $\mu_{0}$, of the reference medium and the latter must be determined numerically. This optimal shear modulus, which leads to the lowest number of iterations at convergence of the iterative scheme is generally strongly influenced by the local material properties. In the present case, the optimal value of $\mu_{0}$ depends on $\sigma_{0}$ and on the power-law exponent $m$. Therefore, the optimal value of $\mu_{0}$ is determined numerically on a lower grid of $32 \times 32$ pixels, for which the solution of the cell problem is very fast. The optimal reference medium has been determined for one occurrence with $M=15$ inclusions. The optimal value obtained for $\mu_{0}$ is thereafter considered for computing the solution in all other cases. This numerical research of the optimal value is illustrated on figures 4 and 5 . On figure 4 we represent the number of iterations at convergence of the E-polarization scheme as a function of $\mu_{0} /\left(\sigma_{0} / 3\right)$ for $m=0.5$. Figure 4 displays similar results for $m=0.2$. On each figure the full line represents the number of iterations obtained with a grid of $512 \times 512$ pixels while the dashed curve is obtained with a grid of $32 \times 32$ pixels. It can be observed that the optimal reference medium (corresponding to the minimum of each curve) is relatively independent on the resolution of the image.

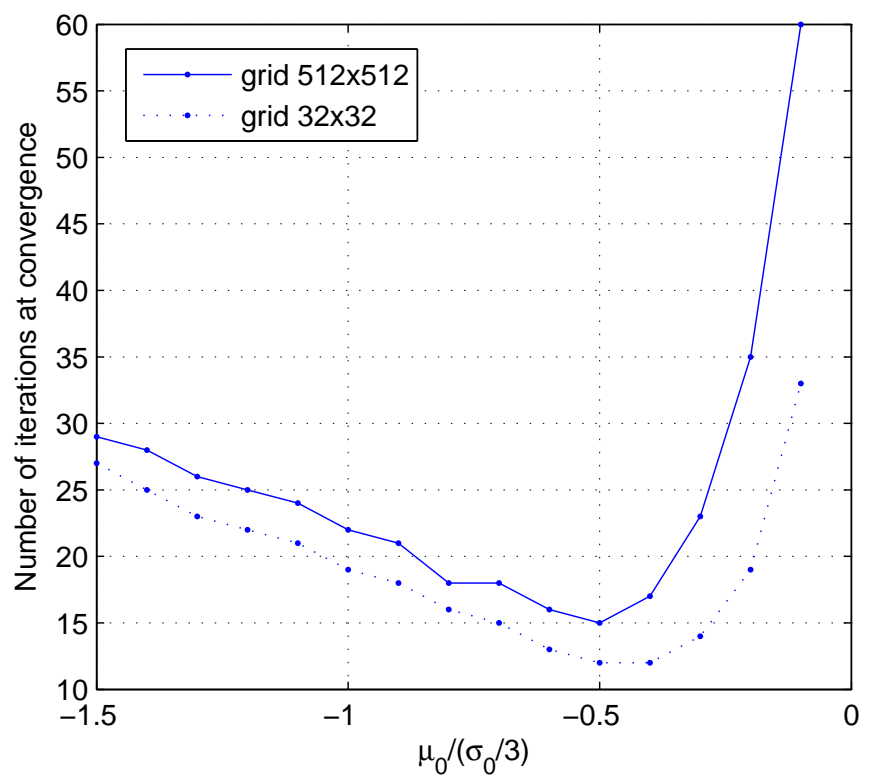

Fig. 4. Number of iterations at convergence of the E-polarization scheme as a function of $\mu_{0} /\left(\sigma_{0} / 3\right)$ for $m=0.5$ and for two grids: $32 \times 32$ pixels (dashed line) and $512 \times 512$ pixels (full line). 


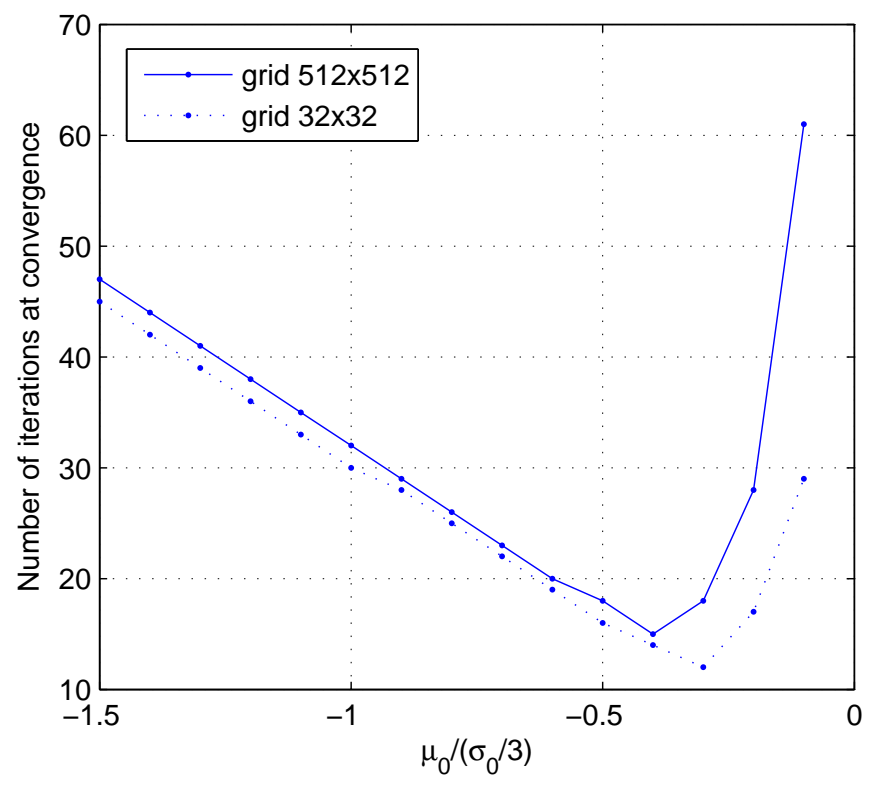

Fig. 5. Number of iterations at convergence of the E-polarization scheme as a function of $\mu_{0} /\left(\sigma_{0} / 3\right)$ for $m=0.2$ and for two grids: $32 \times 32$ pixels (dashed line) and $512 \times 512$ pixels (full line).

On figure 6 we represent the number of iterations at convergence of the Epolarization iterative scheme as a function of the power-law exponent $m$ for various volume fractions of inclusions and different occurences. A good rate of convergence of the iterative scheme is observed in all situations. Moreover it can be noticed that the number of iterations at convergence depends weakly on the power-law exponent. We now present results for the homogenized equivalent stress $\sigma_{0}^{\text {hom }}$ defined by:

$$
\sigma_{0}^{h o m}=\frac{\Sigma_{e q}}{\left(E_{e q}\right)^{m}}
$$

where $\Sigma_{e q}=\sqrt{\frac{3}{2} \bar{\Sigma}: \bar{\Sigma}}$ and $E_{e q}=\sqrt{\frac{2}{3} \overline{\boldsymbol{E}}: \overline{\boldsymbol{E}}}$ are respectively the macroscopic equivalent stress and strain. The macroscopic stress $\Sigma$ is computed such as $\boldsymbol{\Sigma}=<\boldsymbol{\sigma}(\underline{x})>_{V}$ at convergence of the E-polarization based iterative scheme. The cell is still submitted to the macroscopic loading $E_{11}=-E_{22}=1$ and $E_{12}=0$. The effective behavior of the non linear composite with a random distribution of circular inclusions is evaluated by taking the average of the equivalent stress obtained for a number of different occurrences. For instance, on figure 7 , we represent the value of $\sigma_{0}^{\text {hom }} / \sigma_{0}$ for various occurrences and for the volume fraction of inclusions $c_{I}=0.118$ corresponding to $M=15$ inclusions randomly distributed in the cell. The discrete points (the stars) represent the value of $\sigma_{0}^{\text {hom }} / \sigma_{0}$ computed by the FFT algorithm while the full line represent the cumulated mean value of $\sigma_{0}^{\text {hom }} / \sigma_{0}$. For each volume fraction, 
the mean value of $\sigma_{0}^{\text {hom }} / \sigma_{0}$ is then evaluated with 100 occurrences. For more than 100 iterations, one observes that the mean value of $\sigma_{0}^{\text {hom }} / \sigma_{0}$ oscillates in a range inferior to 0.1 percents.

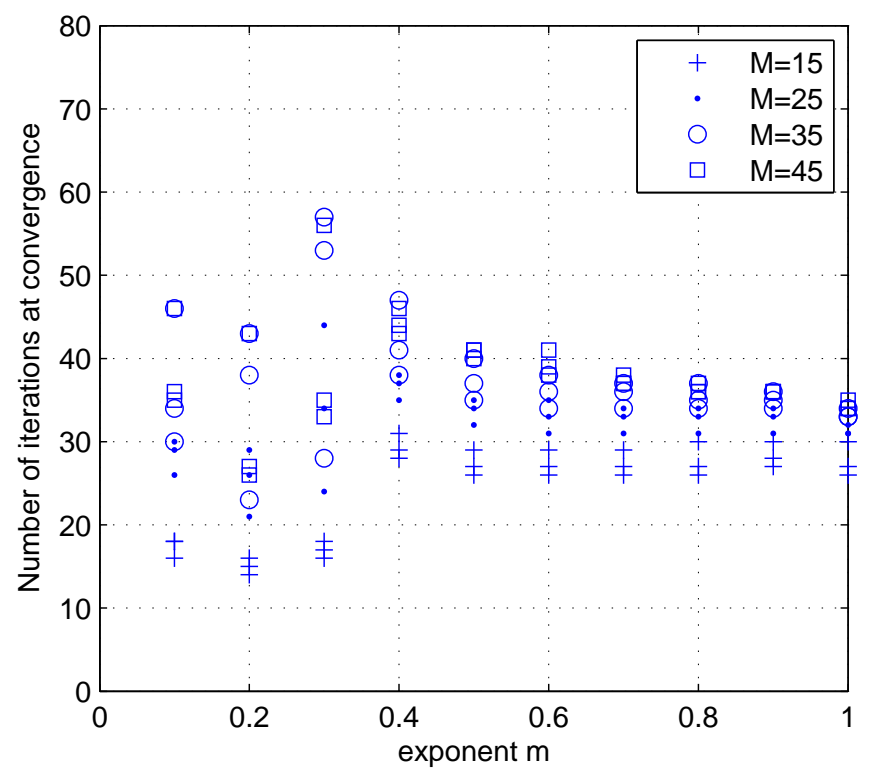

Fig. 6. Number of iterations at convergence of the E-polarization iterative scheme as a function of the power law exponent for various volume fractions of the inclusions (corresponding to $M=15,25,35,45$ inclusions in the cell) and occurences.

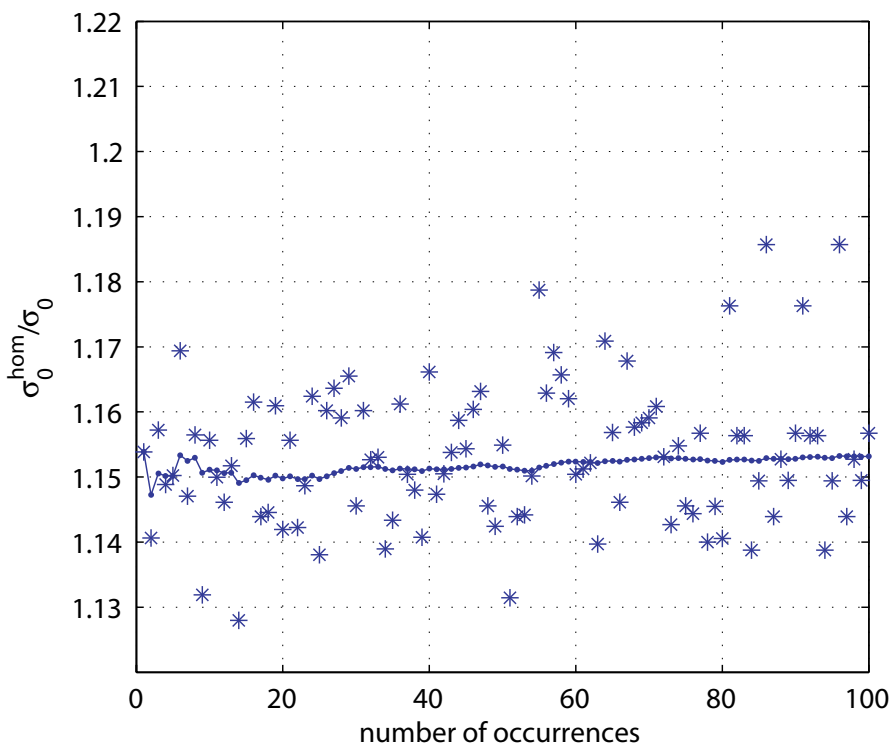

Fig. 7. Value of the homogenized equivalent stress $\sigma_{0}^{\text {hom }} / \sigma_{0}$ for various occurences (the stars). Cumulated mean value of $\sigma_{0}^{\text {hom }} / \sigma_{0}$ as a function of the number of occurences. 
On figure 8, we represent the homogenized effective stress $\sigma_{0}^{\text {hom }} / \sigma_{0}$ as a function of the power-law exponent for a volume fraction $c_{I}=0.118$. The results obtained by the FFT iterative scheme are compared with analytic expressions obtained from non linear homogenization methods provided in $[6,23,26,24]$, see also [16] for a synthesis of these results in the case of power-law matrix reinforced by rigid inclusions. On figure 8, the FFT results are then compared with the variational estimate based on the Hashin-Shtrikman bound "VE(HS)", the second order estimate using the Hashin Shtrikman Bound "SOE(HS-)" and the second order estimate based on the self consistent scheme "SOE(SC)". Figure 9, 10 and 11 give similar results for the three other values of the volume fraction of inclusions.

It can be noticed that the estimates based on the Hashin-Shtrikman bound "VE(HS-)" and "SOE(HS-)" are not very accurate in the case of a random distribution of circular inclusions. In another hand, the second order estimate based on the self consistent scheme "SOE(SC)" is in a good agreement with our FFT based results for low values of the volume fraction of rigid inclusions corresponding to figures 8 and 9. This estimate is less accurate for higher values of porosity, corresponding to figures 10 and 11 .

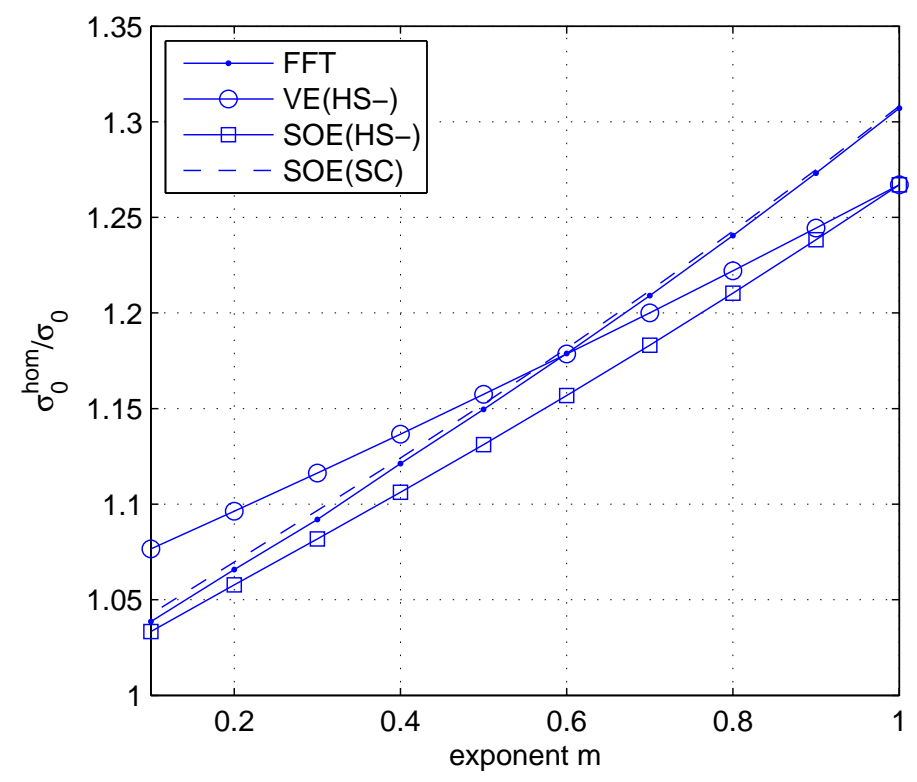

Fig. 8. Value of the homogenized equivalent stress $\sigma_{0}^{\text {hom }} / \sigma_{0}$ as a function of the power-law exponent for a volume fraction $c_{2}=0.118$ ( $M=15$ inclusions). Comparison of the FFT results with analytic expressions obtained from non linear homogenization methods. 


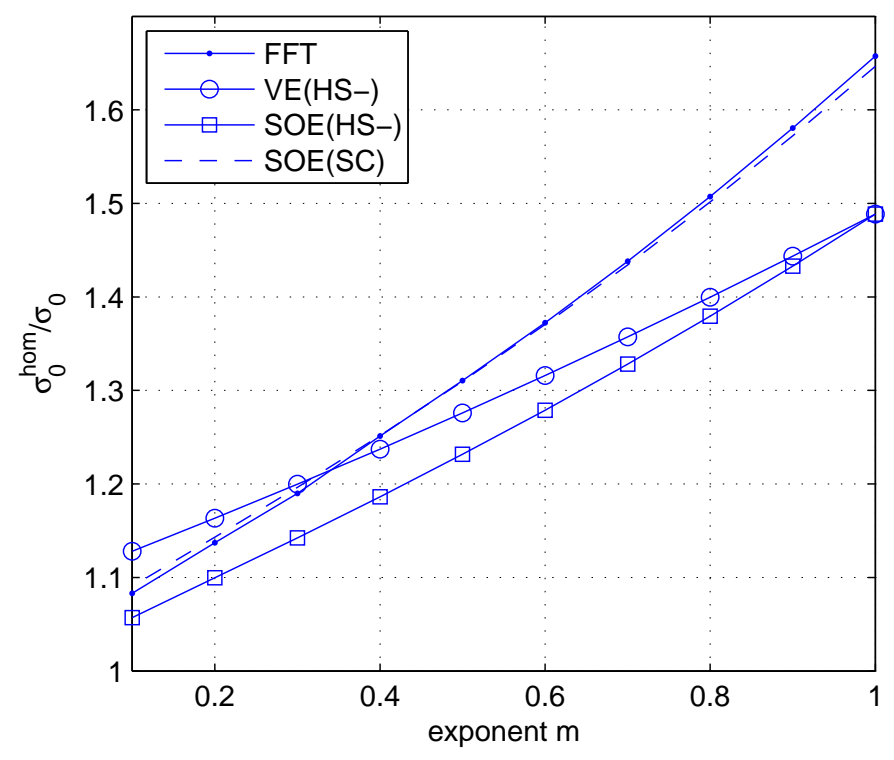

Fig. 9. Value of the homogenized equivalent stress $\sigma_{0}^{\text {hom }} / \sigma_{0}$ as a function of the power-law exponent for a volume fraction $c_{2}=0.196$ ( $M=15$ inclusions). Comparison of the FFT results with analytic expressions obtained from non linear homogenization methods.

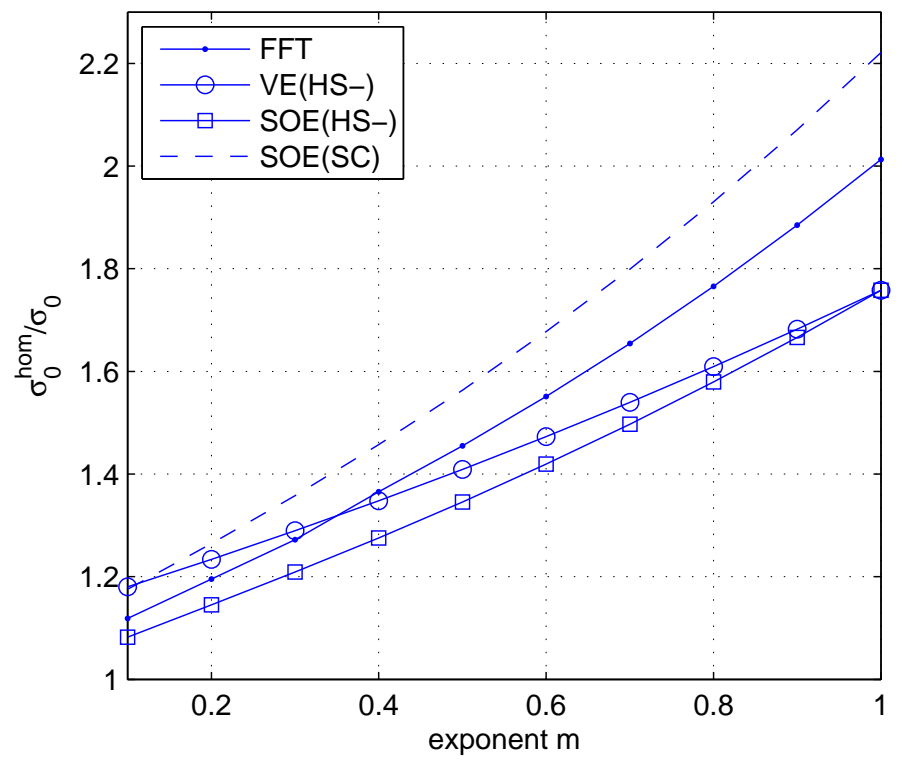

Fig. 10. Value of the homogenized equivalent stress $\sigma_{0}^{\text {hom }} / \sigma_{0}$ as a function of the power-law exponent for a volume fraction $c_{2}=0.275$ ( $M=15$ inclusions). Comparison of the FFT results with analytic expressions obtained from non linear homogenization methods. 


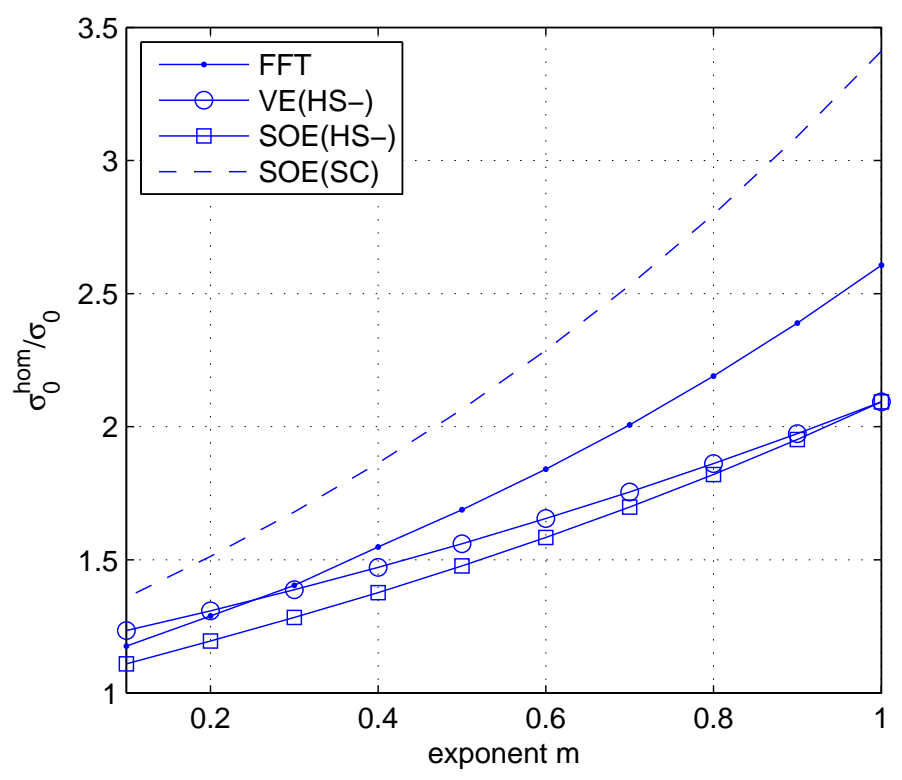

Fig. 11. Value of the homogenized equivalent stress $\sigma_{0}^{\text {hom }} / \sigma_{0}$ as a function of the power-law exponent for a volume fraction $c_{2}=0.353$ ( $M=15$ inclusions). Comparison of the FFT results with analytic expressions obtained from non linear homogenization methods.

\subsection{Exemple 2: macroscopic yield surface of porous materials}

In this second example we propose to determine the macroscopic yield surface of porous material containing cylindrical cavities. The problem is two dimensional, the unit cells of the porous medium are given on figures 3 . Plane stress conditions are considered and the basic cell is subjected to the following macroscopic strain loadings:

$$
E_{i j}=\left(\begin{array}{ccc}
E_{11} & 0 & 0 \\
0 & E_{22} & 0 \\
0 & 0 & 0
\end{array}\right), \quad E_{i j}=\left(\begin{array}{ccc}
E_{11} & E_{12} & 0 \\
E_{12} & E_{11} & 0 \\
0 & 0 & 0
\end{array}\right)
$$

The methodology for the determination of the macroscopic yield stress is inspired of one already proposed in [16] and also used in [2]. Since for rigid ideally plastic material the strain energy (in fact the dissipation potential) is a non smooth function of the strain, the idea is to use the following differentiable expression: 


$$
\begin{aligned}
& w(\varepsilon(\underline{x}))=\frac{9}{2} k \varepsilon_{m}(\underline{x})^{2}+f\left(\varepsilon_{e q}(\underline{x})\right) \\
& f\left(\varepsilon_{e q}(\underline{x})\right)=\left\{\begin{array}{lr}
\frac{3}{2} \mu \varepsilon_{e q}(\underline{x})^{2}+\frac{\sigma_{0}^{2}}{6 \mu} & \text { if } \varepsilon_{e q}<\varepsilon_{0} \\
\sigma_{0} \varepsilon_{e q} & \text { if } \varepsilon_{e q} \geq \varepsilon_{0}
\end{array}\right.
\end{aligned}
$$

with:

$$
\varepsilon_{e q}=\sqrt{\frac{2}{3} \bar{\varepsilon}: \bar{\varepsilon}}, \quad \varepsilon_{0}=\frac{\sigma_{0}}{3 \mu}
$$

and $\varepsilon_{0}=\sigma_{0} /(3 \mu)$. In (19), $k$ and $\mu$ are the elastic bulk and shear moduli of the matrix. $\sigma_{0}$ is the yield stress in uniaxial tension of the matrix. The introduction of elastic coefficient does not affect the value of the macroscopic yield strength but is just a numeric artifice in order to make the function differentiable at the origin. By derivation, the strain-stress relation in the matrix is:

$$
\overline{\boldsymbol{\sigma}}= \begin{cases}2 \mu \overline{\boldsymbol{\varepsilon}} & \text { if } \varepsilon_{e q}<\varepsilon_{0} \\ \frac{2 \sigma_{0}}{3 \varepsilon_{e q}} \bar{\varepsilon} & \text { if } \varepsilon_{e q} \geq \varepsilon_{0}\end{cases}
$$

The polarization is $\boldsymbol{\tau}(\underline{x})=\tau_{m}(\underline{x}) \boldsymbol{I}+\overline{\boldsymbol{\tau}}(\underline{x})$ with:

$$
\tau_{m}(\underline{x})=3\left(k-k_{0}\right) \varepsilon_{m}(\underline{x})
$$

while the deviatoric part is:

$$
\overline{\boldsymbol{\tau}}(\underline{x})=\overline{\boldsymbol{\sigma}}-2 \mu_{0} \overline{\boldsymbol{\varepsilon}}= \begin{cases}2\left(\mu-\mu_{0}\right) \overline{\boldsymbol{\varepsilon}} & \text { if } \varepsilon_{e q}<\varepsilon_{0} \\ 2\left(\frac{\sigma_{0}}{3 \varepsilon_{e q}}-\mu_{0}\right) \overline{\boldsymbol{\varepsilon}} & \text { if } \varepsilon_{e q} \geq \varepsilon_{0}\end{cases}
$$

Let us now express the strain has function of the polarization. The inversion of (22) is trivial:

$$
\varepsilon_{m}(\underline{x})=\frac{\tau_{m}(\underline{x})}{3\left(k-k_{0}\right)}
$$

The inversion of (23) for $\varepsilon_{e q}<\varepsilon_{0}$ is also trivial: 


$$
\bar{\varepsilon}=\frac{\overline{\boldsymbol{\tau}}(\underline{x})}{3\left(\mu-\mu_{0}\right)}
$$

Consider now the case $\varepsilon_{e q} \geq \varepsilon_{0}$ in (23), we have:

$$
\tau_{e q}=\sqrt{\frac{3}{2} \overline{\boldsymbol{\tau}}: \overline{\boldsymbol{\tau}}}=\sigma_{0}-3 \mu_{0} \varepsilon_{e q}
$$

In particular, for $\varepsilon_{e q}=\varepsilon_{0}$, one has:

$$
\tau_{e q}=\sigma_{0}-3 \mu_{0} \varepsilon_{0}=\sigma_{0}\left(1-\frac{\mu_{0}}{\mu}\right)
$$

Let us introduce $\tau_{0}$, defined by:

$$
\tau_{0}=\sigma_{0}\left(1-\frac{\mu_{0}}{\mu}\right)
$$

The deviatoric part of the strain is then given by:

$$
\overline{\boldsymbol{\varepsilon}}=\left\{\begin{array}{l}
\frac{\overline{\boldsymbol{\tau}}}{2\left(\mu-\mu_{0}\right)} \text { if } \tau_{e q}<\tau_{0} \\
\frac{\sigma_{0}-\tau_{e q}}{2 \mu_{0} \tau_{e q}} \overline{\boldsymbol{\tau}} \text { if } \tau_{e q} \geq \tau_{0}
\end{array}\right.
$$

In the cavity the stress field being null, the relation giving the strain as function of the polarization is:

$$
\varepsilon=-\mathbb{S}_{0}: \tau(\underline{x})
$$

with:

$$
\mathbb{S}_{0}=\mathbb{C}_{0}^{-1}=\frac{1}{3 k_{0}} \mathbb{J}+\frac{1}{2 \mu_{0}} \mathbb{K}
$$

with $\mathbb{J}=\frac{1}{3} \boldsymbol{I} \otimes \boldsymbol{I}, \mathbb{K}=\mathbb{I}-\mathbb{J}$ where it is recalled that $\boldsymbol{I}$ is the two order identity tensor and $\mathbb{I}$ is the fourth order, symmetric identity tensor.

With relations (24) and (29) and (30), it is possible to compute the strain due to a given polarization, as required in the polarisation based iterative schemes. Again, the $\boldsymbol{E}$-polarization scheme is considered to solve the non linear problem. In our calculations, the resolution $512 \times 512$ is considered, the value is $\sigma_{0}=1$ is chosen for the yield stress of the matrix and the following values for the elastic 
coefficient of the matrix and the reference medium are considered: $\mu=\lambda=1$ and $\mu_{0}=\lambda_{0}=-1$.

The components of the macroscopic strain are chosen on the form:

$$
\begin{aligned}
& E_{11}=q \cos (\theta), \quad E_{22}=q \sin (\theta) \quad 1^{s t} \text { loading case } \\
& E_{11}=q \cos (\theta), \quad E_{12}=q \sin (\theta) \quad 2^{\text {nd }} \text { loading case }
\end{aligned}
$$

where the value of $\theta$ is fixed and the value of $q$ is applied incrementally. It is then possible to reproduce the macroscopic strain stress curve and, for large values of $q$, to determine the values of macroscopic stress that stands on the macroscopic surface (the author could refers to [2] for more details). Various values of the angle $\theta$ are considered in the range $[0, \pi / 2]$ in order to reproduce the yield surface in the plane $\Sigma_{h}-\Sigma_{e q}$ with $\Sigma_{h}=\left(\sigma_{11}+\Sigma_{22}\right) / 2$. In each cases, the unit cell is squared and represented on figure (3) (first image on the left side). The volume fraction of inclusions is $c_{I}=0.118$ and the number of inclusions is $M=15$.

On figure 12 we represent the macroscopic criterion in the plane $\Sigma_{h}-\Sigma_{e q}$. On this figure we compare the FFT simulations with the Gurson criterion [11]:

$$
\frac{\Sigma_{e q}^{2}}{\sigma_{0}^{2}}+2 f \cosh \left(\frac{\sqrt{3}}{2} \frac{\Sigma_{11}+\Sigma_{22}}{\sigma_{0}}\right)-1-f^{2}=0
$$

and the criterion obtained by [19]:

$$
\frac{\Sigma_{e q}^{2}}{\sigma_{0}^{2}}+2 f \cosh \left\{\frac{1}{\sigma_{0}} \sqrt{\frac{3}{2}\left(\Sigma_{11}^{2}+\Sigma_{22}^{2}\right)+3 \Sigma_{12}^{2}+3 \Sigma_{13}^{2}+3 \Sigma_{23}^{2}}\right\}-1-f^{2} \leq 0
$$

Figure 13 displays similar results for the 2nd loading case. It must be noted that the criteria (33) and (34) which comes from the limit analysis of the hollow cylinder overestimates the extremal surface of randomly voided materials. This has been already reported in [16,2] and is attributed to the highly localized plastic deformation which occurs in the ligament between the voids. It can be also observed that the FFT simulations predict a yield surface which is different for the two loading cases while the analytic criteria (33) and (34) produce the same curve in the plane $\Sigma_{h}-\Sigma_{e q}$. The reason is that the analytic criterion are based on the limit analysis of a hollow cylinders submitted to homogeneous strain rate boundary conditions for the Gurson model and homogeneous stress boundary conditions in [19]. So, due to cylindrical geometry of the cell, the resulting analytic criteria for the porous plastic material are equivalent under in plane pure shear stress loading at $0^{\circ}$ or $45^{\circ}$. The differences observed with the FFT simulations between figures 12 and 13 is then attributed to the spatial repartitions of the voids. 


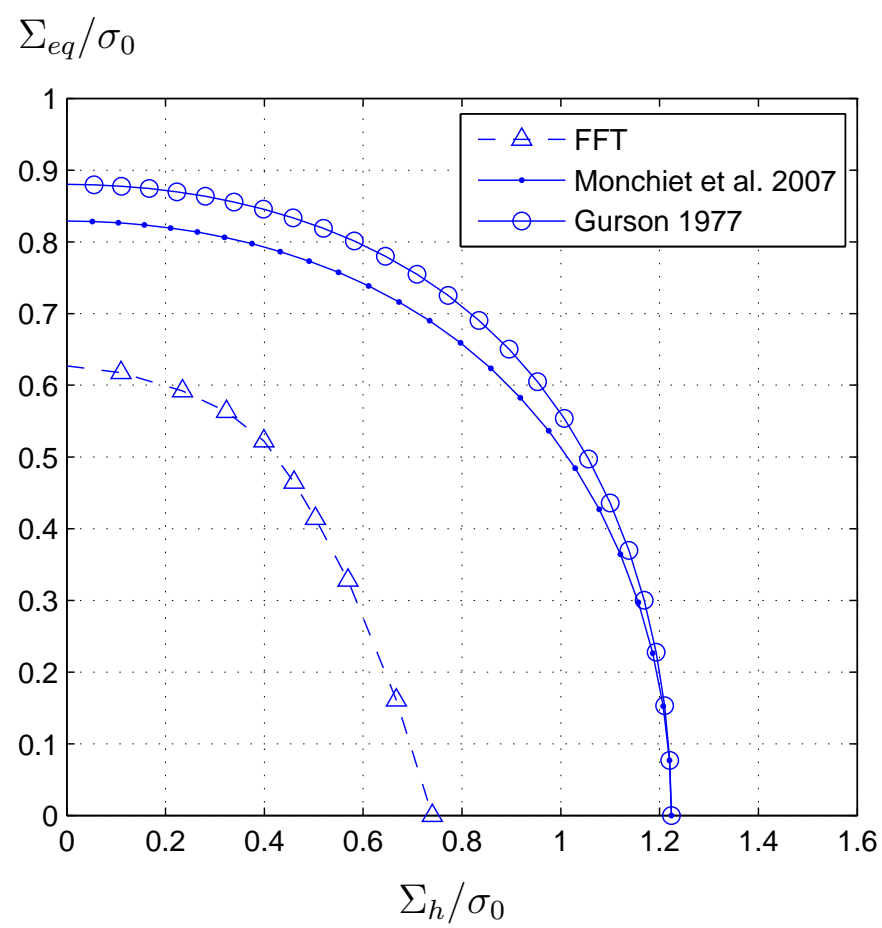

Fig. 12. Macroscopic yield surface for porous material containing randomly distributed cylindrical cavities: 1st loading case. Comparison between FFT simulations, the Gurson criterion [11] and the one of Monchiet et al. [19].

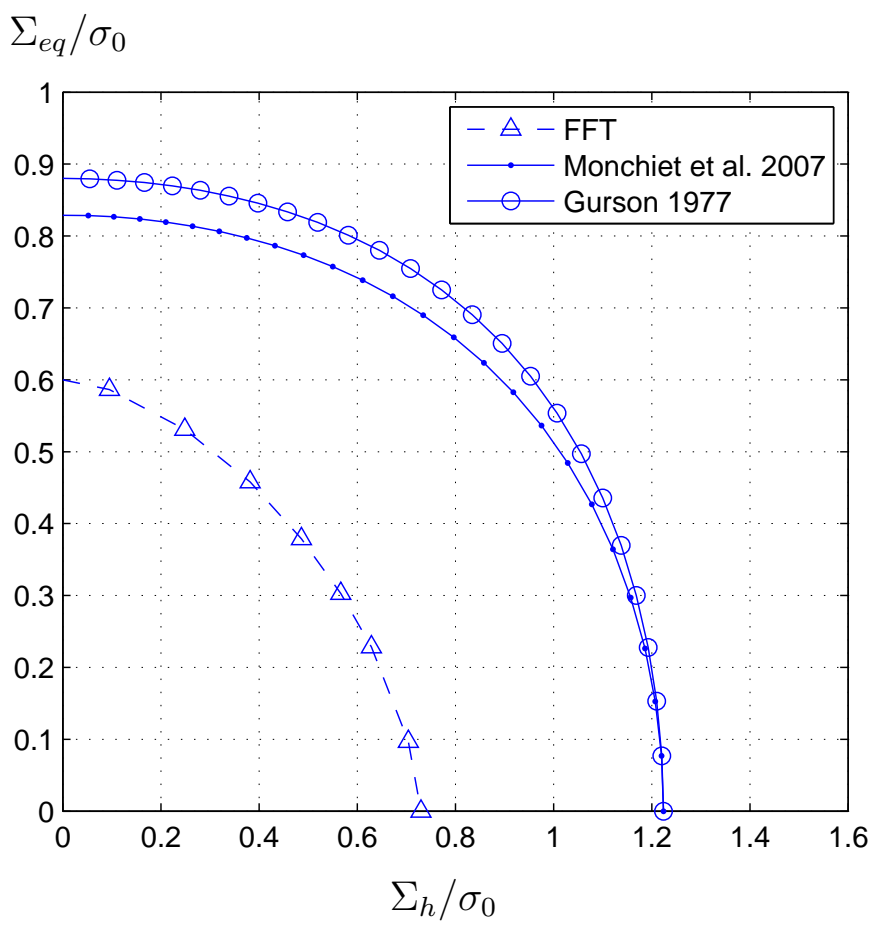

Fig. 13. Macroscopic yield surface for porous material containing randomly distributed cylindrical cavities: 2nd loading case. Comparison between FFT simulations, the Gurson criterion [11] and the one of Monchiet et al. [19]. 


\section{Conclusion}

This paper has dealt with an extension of the polarization based iterative scheme in the context of non linear composites. This approach performs the computation of the solution of the cell problem by means of an iterative scheme which uses exact expressions of the Green operators in Fourier space and the Fast Fourier Transform (FFT) algorithm which makes the computation very fast. One specificity of the polarization approach initially proposed in [17] was to provide the solution of an auxiliary problem for which an average polarization is submitted to the unit cell of the periodic composite. In order to deal with non linear composites we have provided some modifications which allow to deal with prescribed macroscopic strains or stresses. The rate of convergence of these two new algorithms, called E-polarization scheme and $\Sigma$-polarization scheme, has been first evaluated in the case of linear elastic constituents. It has been shown that the convergence of these two new approaches is as efficient as the one of the original polarization scheme. The E-polarization iterative scheme has been next extended in the context of non linear composites. The example considered in the paper consists of a power-law type non linear elastic matrix containing rigid inclusions which are randomly distributed in the unit cell. Again a good convergence of this algorithm has been observed and this, whatever the values of the power-law exponent. The homogenized effective stress has been computed and compared with analytic expressions obtained in the framework of non linear homogenization methods. As a second example, we have applied the new iterative scheme for the determination of the macroscopic yield surface of plastic materials materials containing randomly distributed cylindrical cavities. The results have been compared to available micromechanics based analytic expressions of the macroscopic criteria of ductile porous material.

\section{References}

[1] K. Bhattacharya and P. Suquet. A model problem concerning recoverable strains of shape-memory polycrystals. Proc. Roy. Soc. London A., 461:2797281,2005 .

[2] N. Bilger, F. Auslender, M. Bornert, J.-C. Michel, H. Moulinec, P. Suquet, and A. Zaoui. Effect of a nonuniform distribution of voids on the plastic response of voided materials: a computational and statistical analysis. Int. J. Solids Struct., 42:517-538, 2005.

[3] G. Bonnet. Effective properties of elastic periodic composite media with fibers. J. Mech. Phys. Solids, 55:881-899, 2007. 
[4] R. Brenner. Numerical computation of the response of piezoelectric composites using Fourier transform. Phys. Rev., B 79:184106, 2009.

[5] R. Brenner, R.A. Lebensohn, and O. Castelnau. Elastic anisotropy and yield surface estimates of polycrystals. Int. J. Sol. Struct., 46:3018-3026, 2009.

[6] J.R. Willis D. Talbot. Variational principles for inhomogeneous nonlinear media. IMA Journal of Applied Mathematics, 35:39-54, 1985.

[7] D.J Eyre and G.W. Milton. A fast numerical scheme for computing the response of composites using grid refinement. J. Phys. III, 6:41-47, 1999.

[8] F. Feyel. Multiscale FE2 elastoviscoplastic analysis of composite structure. Comput. Mater. Sci., 16(1Ü4):433-454, 1999.

[9] S. Ghosh, K. Lee, and P. Raghavan. A multilevel computational model for multi-scale damage analysis in composite and porous media. Int. J. Sol. Struct., $38: 2335-2385,2006$.

[10] E. Ghossein and M. Lévesque. A fully automated numerical tool for a comprehensive validation of homogenization models and its application to spherical particles reinforced composites. Int. J. Sol. Struct., 49:1387-1398, 2012 .

[11] A.L. Gurson. Continuum theory of ductile rupture by void nucleation and growth. part i. - yield criterion and flow rules for porous ductile media. $J$. Engrg. Mater. Technology., 99:2-15, 1977.

[12] R.A. Lebensohn, A.K. Kanjarla, and P. Eisenlohr. An elasto-viscoplastic formulation based on fast Fourier transforms for the prediction of micromechanical fields in polycrystalline materials. Int. J. Plasticity, 32-33:5969, 2008.

[13] S.B. Lee, R.A. Lebensohn, and A.D. Rollett. Modeling the viscoplastic micromechanical response of two-phase materials using Fast Fourier Transforms. Int. J. Plasticity, 27(5):707-727, 2010.

[14] J. Li, S. Meng, X. Tian, F. Song, and C. Jiang. A non-local fracture model for composite laminates and numerical simulations by using the FFT method. Composites: Part B, 43:961-971, 2012.

[15] J.-C. Michel, H. Moulinec, and P. Suquet. A computational method based on augmented lagrangians and fast Fourier transforms for composites with high contrast. Comput. Model. Eng. Sci., 1(2):9-88, 2000.

[16] J.-C. Michel, H. Moulinec, and P. Suquet. A computational scheme for linear and non-linear composites with arbitrary phase contrast. Int. J. Numer. Meth. Engrg., 52:139-160, 2001.

[17] V. Monchiet and G. Bonnet. A polarization-based FFT iterative scheme for computing the effective properties of elastic composites with arbitrary contrast. Int. J. Num. Meth. Engrg., 89(11):1419-1436, 2012. 
[18] V. Monchiet, G. Bonnet, and G. Lauriat. A FFT-based method to compute the permeability induced by a Stokes slip flow through a porous medium. $C$. R. Méca., 337(4):192-197, 2009.

[19] V. Monchiet, E. Charkaluk, and D. Kondo. An improvement of gurson-type models of porous materials by using eshelby-like trial velocity fields. $C . R$. Méca., 335(1):32-41, 2007.

[20] H. Moulinec and P. Suquet. A fast numerical method for computing the linear and nonlinear mechanical properties of composites. C. R. Acad. Sci., 318(11):1417-1423, 1994.

[21] H. Moulinec and P. Suquet. A numerical method for computing the overall response of nonlinear composites with complex microstructure. Comput. Meth. Appl. Mech. Engrg., 157:69-94, 1998.

[22] T.-K. Nguyen, V. Monchiet, and G. Bonnet. A fourier based numerical method for computing the dynamic permeability of porous media. Eur. J. Mech. B/fluids, (DOI: 10.1016/j.euromechflu.2012.07.004), 2012.

[23] P. Ponte-Castañeda. The effective mechanical properties of nonlinear isotropic composites. J. Mech. Phys. Solids, 39:45-71, 1991.

[24] P. Ponte-Castañeda and P. Suquet. Nonlinear composites. Adv. Appl. Mech, 34:171-302, 1998.

[25] R. Smit, W. Brekelmans, and H. Meijer. Prediction of the mechanical behavior of nonlinear heterogeneous systems by multi-level finite element modeling. Comput. Meth. Appl. Mech. Engrg., 155:181-192, 1998.

[26] P. Suquet. Overall potentials and extremal surfaces of power law or ideally plastic materials. Journal of the Mechanics and Physics of Solids, 41:981-1002, 1993.

[27] V. Vinogradov and G.W. Milton. An accelerated fft algorithm for thermoelastic and non-linear composites. Int. J. Num. Meth. Engrg., 76(11):1678-1695, 2008.

[28] F. Willot and D. Jeulin. Elastic behavior of composites containing boolean random sets of inhomogeneities. Int. J. Engrg. Science, 47:313-324, 2009.

[29] J. Yvonnet. A fast method for solving microstructural problems defined by digital images: a space Lippman-Schwinger scheme. Int. J. Num. Meth. Engrg., DOI: $10.1002 /$ nme.4334, 2012. 